\title{
Working Memory Performance of Expert and Novice Interpreters
}

\author{
Migyong Lee \\ Kyung Hee University
}

\begin{abstract}
As in any field of specialization, simultaneous interpreting requires expertise which distinguishes the conference interpreters from other bilinguals. Involved in coordination and regulation of ongoing processing of information, working memory may be one of the factors that constitute the expertise of conference interpreters. The aim of this study is to investigate the working memory performance of interpreters and the effect of their working memory performance on the capacity to mediate communication under time constraints. Under such an assumption, the working memory spans of two groups of conference interpreters - seven interpreters in the Experienced Group with more than ten years of working experience and sixteen interpreters in the Novice Group with less than three years of experience - were measured. In addition, a survey was conducted to assess the topic knowledge of the two groups of interpreters. English into Korean simultaneous interpreting experiment was carried out to investigate the implications of
\end{abstract}

Migyong Lee

English Department Kyung Hee University

1 Hoegi-dong, Dongdaemun-ku Seoul, Korea 130-701

Phone: 822-961-0224; Email: migyonglee@khu.ac.kr

Received Jan. 2011; Reviewed Feb. 2011; Revised version received Feb. 2011. 
96 Working Memory Performance

working memory performance on the interpreters' ability to transfer information from the source language to the target language. The interpreters' ability to transfer information was measured in terms of their ability to transfer meaning segments referred to as Idea Units and Essential Idea Units in the texts. The results of the experiment showed that the Experienced Group was able to transfer a higher percentage of both Idea Units and Essential Idea Units than the Novice Group. There was higher correlations between the Novice Group of interpreters' working memory performance and their ability to transfer information while higher correlations was seen between topic knowledge and ability to transfer information among the Experienced Group of interpreters.

Keywords: working memory performance, expertise, simultaneous interpreting, information processing

\section{Introduction}

The difficulties in the realization of the communicative act in Simultaneous Interpreting (SI) arise mainly from the unique features of this mode of communication. The activity of SI is heavily marked by the temporal load. Whereas translators of written texts have ample time to search for the most accurate terms and to express themselves in the best possible style, the interpreter's choices are severely restricted in the process of converting oral messages at a speed which is about thirty times faster than that of the translator (Seleskovitch 1978). Another salient characteristic of the SI task is its multitasking complexity. At any given point in time, the interpreter has to comprehend and translate the Source-language Text (ST), as well as formulate, produce and monitor their Targetlanguage Text (TT) all while keeping track of the coherence of the original, the accuracy of their translation, the smoothness of the delivery, among others (Gerver 1976; Seleskovitch \& Lederer 1982; Jones 1998). 
In spite of the enormous complexity of the interpreting task, it is a commonplace to see interpreters doing it routinely. Interpreters in fact perform very well at ST presentation rate of 120 words per minute (WPM). Interpreters can perform with $90 \%$ accuracy, while speaking and listening simultaneously $65 \%$ to $75 \%$ of the time (Barik 1975). The fact that interpreting is so routinely carried out in spite of its complexity raises a question: how do professional interpreters carry out the SI smoothly and efficiently? That is, what constitutes the interpreting skills that sets them apart from other bilinguals or trilinguals? Riccardi (1988) remarked, "Many of the strategies employed during the course of protracted and demanding cognitive activities may be considered devices for easing the burden, in other words, ways of minimizing cognitive strain." Setton (2003) noted as a way of alleviating cognitive load under time constraint, interpreters would reduce depth of processing, and in particular bypass some semantic processing in favor of direct language-tolanguage translation. Gile (1995) emphasized the importance of balancing and allocating available efforts to suffice listening, analysis, memory and production requirements.

The existing literatures suggest, among others, two factors distributing to overcome constraints inherent in this mode of communication. One is knowledge about the topic being discussed (Seleskovitch \& Lederer 1982; Jones 1998) and the other is working memory capacity which control efficient and skillful allocation of attentional resources (Daneman \& Carpenter 1980). This study endeavors to focus on these two factors: the interpreter's topic knowledge and working memory performance, and how these factors impact the performance of interpreters during SI, specifically in terms of the delivery of information in ST meaning segment to TT. It is hypothesized in this study that topic knowledge and working memory capacity influence the performance of conference interpreters in ways such as how much information is transferred accurately and how the information is expressed. As for the working 
98 Working Memory Performance

memory capacity, the interpreter with a high working memory span will be able to better distribute attentional resources and deliver ST information with high accuracy. On the other hand, profound topic knowledge will enable interpreters to choose shortcuts in the delivery by selecting information with higher priority.

The experiments were designed to measure the working memory capacity of two groups of interpreters: the Novice Group and Experienced Group. Also, the prior topic knowledge of these two groups of interpreters was surveyed. Then, the SI performances of the interpreters interpreting English into Korean were analyzed in terms of the percentage in accuracy in the delivery of information in meaning segments. Then the percentages of information delivered by respective groups were compared to their working memory capacity and topic knowledge.

\section{Interpreters' Topic Knowledge vs. Working Memory}

\subsection{Topic Knowledge}

According to Dillinger (1994), comprehension in interpreting is not a specialized ability but the application of an existing skill under more unusual circumstances. Comprehension in interpreting is characterized by all the same component processes as listening, with an emphasis on semantic processing, in particular proposition generation. He concludes from this that interpreter performance is limited by the same broad parameters that limit text comprehension in general, that is, the nature of the text itself and the prior knowledge that interpreters can bring to bear on understanding it. Unless the interpreter is party to the extralinguistic and situational knowledge assumed by the speaker, has been given advance access to the text which the speaker is about to deliver and is able to refer 
to it throughout, she must allow a certain amount of time to elapse between the point at which a unit of input reaches her ear and the moment when she reproduces it in the TT. The longer this EarVoice Span will force the interpreter to verbalize the TT message more economically. On the other hand, topic knowledge tantamount to a specialist or speaker will allow for not only more room to flexibly re-express ST message into TT, but also select information with high priority.

\subsection{Survey of Topic Knowledge}

The topic knowledge of the two groups of interpreters was analyzed using a survey that assesses the overall familiarity of topic in a 1 to 10 scale. The survey included questions on the topics as well as questions on the level of ease and confidence the interpreters felt about the topics. In addition, the frequency and number of working years related to the topic were evaluated. Higher number of interpreters in Experienced Group had higher number of years working at conferences on the relevant topics. The Experienced Group, in general, felt more confident and at ease about their performances and the speed of ST input. The distribution of points given to each interpreter is shown in Table 1.

Table 1. Assessment of Topic Knowledge

\begin{tabular}{|l|l|}
\hline I. Working experience & \\
\hline+10 yrs & 4 points \\
\hline-5 yrs & 2 points \\
\hline II. Number of days working per year & \\
\hline+100 days & 6 points \\
\hline $50-100$ days & 4 points \\
\hline $1-50$ days & 2 points \\
\hline III. Knowledge about topic discussed & $1-10$ points \\
\hline
\end{tabular}


100 Working Memory Performance

\subsection{Working Memory}

Working memory is generally considered to be both a computational and storage workplace for currently active information (Daneman \& Carpenter 1980; Just \& Carpenter 1992). Just \& Carpenter (1992) proposed that working memory for language is both a pool of limited cognitive resources that supports language processing and a temporary storage of the products of such processing. In proposing a "capacity theory of comprehension," Just $\&$ Carpenter (1992) discussed the limitations that working memory capacity placed on undertaking complex operations such as SI. They purport that the components of working memory, visuospatial sketchpad and phonological loop, as well as central executive all share the limited cognitive resource. Thus, when the load on processing increases, there will be less to go around to store information or carry on other tasks. Any shortage in the function of storage can result in loss or forgetting of information, while shortage in the processing function will result in a slow-down in processing (Carpenter, Miyake \& Just 1994). In the views of Just \& Carpenter, how the limited resources in working memory are deployed can have a significant effect on cognitive performance. Individual differences in working memory capacity will influence the points at which trade-offs occur between processing and storage that are necessary for carrying out SI. As an interpreter gains experience and training in a field, the efficiency in the use of working memory resources to undertake the tasks in that field will improve.

\subsection{Measurement of Working Memory Performance}

Simple but reliable working memory tasks were designed and administered to two groups of 23 interpreters to measure their working memory span. The aim was to investigate how the working memory capacities of interpreters with different years of working 
experiences differ and whether such difference can be ascribed as the result of practice and work experience. The study attempted to investigate how the limited cognitive resources of working memory need to be shared for the tasks of storage and information processing as well as for attention shifting during operations similar to SI operation.

Four working memory tasks: listening span, n-back, divided attention, and task with irrelevant speech information (ISE), have been administered to interpreters to measure working memory span. These tasks emphasize both the storage and processing of information in working memory as well as divided attention and inhibition of processing irrelevant information. The assumption was that there will be some differences in the working memory span of these interpreters who have been divided into two groups according to their years of experience working as conference interpreters.

The listening span and n-back tasks are commonly used in studies of working memory to examine performance when storage and processing capacities are simultaneously increased. The divided attention task is relevant because it requires that the attentional system or the central executive to divide resources efficiently between two tasks performed simultaneously. The ISE task includes irrelevant information during a complex span task, requiring participants to ignore irrelevant information and direct attention processing to relevant task.

The percentage of recall accuracy of words at the end of the sentences read out was calculated in Listening Span and ISE tasks. As is typical in these tasks, the number of sentences read out increased and number of words interpreters needed to recall increased. For the N-Back Task, the interpreter needed to recall the number she has heard " $n$ " number back among a series of 5 numbers. For the Divided Attention Task, two different types of tasks were given such as solving simple mathematical questions while listening to words and the accuracy in the recall was measured. For the ISE 
102 Working Memory Performance

Task, two different types of information were simultaneously provided e.g. music and speech, or news and speech, while the interpreter needed to recall the words at the end of each sentences heard.

The results suggest that there are no significant group differences in listening span and n-back tasks. However, the Experienced Group of interpreters showed more consistency in performance as a group than the Novice Group. For the N-back Task, the Novice Group showed more accuracy in the processing of information compared to the Experienced Group. There were group differences in the Divided Attention and ISE tasks. In conditions where the interference from irrelevant information was greater, group difference increased with interference.

Table 2. Results for Working Memory Tasks

\begin{tabular}{lccc}
\hline \multicolumn{1}{c}{ Task } & Mean & SD & Range \\
\hline Listening Span & & & \\
Novice & 3.31 & 0.29 & $2.1-2.5 \sim 5.1-5.5$ \\
Experienced & 2.79 & 0.41 & $3.1-3.5 \sim 4.1-4.5$
\end{tabular}

Results for Working Memory Tasks

\begin{tabular}{llll}
\hline \multicolumn{1}{c}{ Task } & Mean & SD & \multicolumn{1}{c}{ Range } \\
\hline N-Back & & & \\
Novice & 2.13 & 0.24 & $0-1 \sim 4-5$ \\
Experienced & 4.14 & 0.22 & $0-1 \sim 6-10$
\end{tabular}

Results for Working Memory Tasks

\begin{tabular}{cccc}
\hline Task & Mean & SD & Range \\
\hline
\end{tabular}

\section{Divided Attention}

Novice

0.88

0.38

$0-1 \sim 2-3$

Experienced

1.64

0.29

$0-1 \sim 2-3$ 


\section{Transfer of Information During SI}

\subsection{Experiment}

In order to investigate the implications of interpreters' working memory capacity and topic knowledge on their ability to transfer information during SI, experiments were conducted for a two-fold analysis of the SI performances of the interpreters. First, the SI performances of twenty-three interpreters were analyzed by measuring their delivery of Idea Units and Essential Idea Units. Second, the measurements of the delivery of Idea Units and Essential Idea Units were compared with the measurements of these interpreters' working memory capacity as well as their topic knowledge to find out which of the two coincided closely.

Twenty-three professional conference interpreters whose working memory spans were measured were the subjects for the SI performance analysis. Among the participants, there were seven professional conference interpreters with over ten years of experience and 16 professional interpreters with less than three years of experience. The number of working days for each interpreter differed from person to person because some of them were currently employed as in-house interpreters/translators and were not at liberty to take on conference interpreting assignments as others who were freelancing. Their number of working days ranged from less than 50 days to over 120 days. All 23 participants were graduates of the Graduate School of Interpretation and Translation of Hankuk University of Foreign Studies majoring in SI and working as professional interpreters. In the Novice Group, interpreters were working as full-time in-house interpreters $(\mathrm{N}=11)$ or for international institutions or major government ministries, or on a freelance basis $(\mathrm{N}=5)$. In the Experienced Group, the interpreters were all working very actively as freelance interpreters and were also teaching interpretation and translation at educational 
institutions. All participants spoke Korean as their A language and English as their B language, or had comparable first-language competence in speaking both of these languages.

Common to all interpreters was that they had all passed an entrance examination to receive interpretation training, and have all successfully passed the qualifying examination to advance to the second year at the graduate school as Conference Interpretation majors. Lastly, they have passed the Professional Examination to graduate from the course with a MA diploma in Conference Interpretation. All participants in Novice Group were in the age bracket of 25-35, while the Experienced Group was in 35-45 bracket.

In the performance analysis, first, the English into Korean SI performances of professional interpreters in the Experienced Group were compared with that of the Novice Group with less than three years of experience. The purpose of this comparison is two-fold. First, the TT renditions of two groups of interpreters were evaluated using a scoring method to assess the faithfulness and accuracy of their transfer of information in ST to TT. Second, the scores obtained from the analyses were examined to find out whether any correlations can be established between the scores and the working memory capacity of the interpreters and their topic knowledge. For the assessment of the interpreting performances, two criteria were used in the scoring: the percentage of Idea Units (IU) in Critical Sentences delivered correctly and the selection of more relevant information, i.e. Essential Idea Units (EU).

Thirty six Critical Sentences were selected from 4 texts. These sentences, then, were divided into Idea Units and were given one points each. Then, the Idea Units, which contained high priority information (i.e. information that were essential in the delivery of message and must not be omitted or summarized), were given double points. This measuring method was designed in order to investigate whether experienced interpreters are better at not only delivering ST information more accurately, but are able to select 
more important or relevant information to interpret than novices. For this study, Idea Units is defined as meaning units or meaning segments. Units of meaning are segments of sense appearing at irregular intervals in the mind of those who listen to speech with a deliberate desire to understand it (Lederer 1978). Chunks of sense appear in interpretation whenever the interpreter has a clear understanding of a speaker's intended meaning and these meaning units can be preceded by a slight pause. Total number of points given as Idea Units were 234 units while total number of Essential Idea Units were 370 units.

\section{Sample Critical Sentence}

$\overline{\text { ST }}$

Not only/ has Harvard/ given me/ an extraordinary honour,/ but the weeks of fear and nausea I have endured/ at the thought of/ giving this commencement address/ have made me lost weight. IU: 7. EU: 12

TT 1

하버드에서/ 이렇게 연설을 할 수 있게 됨으로서/ 영광을 누리기 도/ 했습니다만/ 이 졸업식 축사를 하기위해서/ 제가/ 몇 달째 굉 장히 괴로웠고요 고민하다 보니까/ 살도 빠졌습니다. IU: 7, EU: 12

TT 2

하버드대학 측에서는/ 저에게 초대를 해 줌으로서/ 일주일동안/ 제가 고민을 했기 때문에/ 살이 빠졌습니다. IU: $5, \mathrm{EU}: 8$

In the example of the above Critical Sentence, the TT1 and TT2 interpreted renditions have been evaluated as having different scores of IU and EU. The TT1 has IU of 7 and EU of 12, while the TT2 has IU of 5 and EU of 8 . This can be interpreted as that the interpreter of TT1 was more faithful to the ST than TT2 in terms of the delivery of the amount of meaning units. Also, interpreter of TT1 delivered more important meaning units from Source Language to Target Language than TT2. 
106 Working Memory Performance

\subsection{Results}

The results show that the Experienced Group of interpreters delivered ST Idea Units more closely and accurately to TT renditions than the Novice Group. The Novice Group of interpreters showed wider differences in performances than the Experienced Group interpreters. It was hypothesized that the Experienced Group will deliver more Idea Units correctly into TT than the Novice Group as an effect of larger working memory capacity. However, the percentage of Idea Units correctly delivered for the Novice Group and Experienced Group were not widely different as shown in the table below. In addition, the texts which the Novice Group showed low performance in the delivery of IU and EU, the Experienced Group also performed poorly. This may be an indication that, although to a different degree, the difficulties in the texts influence not only less experienced interpreters but also experienced interpreters.

Table 3. Delivery of Idea Units \& Essential Idea Units

\begin{tabular}{|c|c|c|c|}
\hline Text & ST & Novice & Experienced \\
\hline \hline Text A & & & \\
I U & 63 & 52.36 & 53.86 \\
E U & 104 & 86.36 & 91.14 \\
\hline Text B & & & \\
I U & 51 & 37.45 & 46.25 \\
E U & 80 & 58.36 & 73.50 \\
\hline Text C & & & \\
I U & 50 & 26.50 & 26.80 \\
E U & 81 & 43.00 & 44.60 \\
\hline Text D & & & \\
I U & 70 & 48.10 & 51.60 \\
E U & 105 & 68.10 & 79.80 \\
\hline \multicolumn{2}{|l}{}
\end{tabular}


For interpreters who work at the saturation threshold of their processing ability, one of the most important effect of expertise would be the ability to decide which information is more relevant and which is secondary. It was hypothesized that the Experienced Group of interpreters will be able to decide more quickly and accurately which Idea Units are more relevant and essential to interpret and which are secondary as the effect of the years of experience as well as topic knowledge.

As shown in Table 7, the gap between the delivery of Essential Idea Units between the Novice Group and Experienced Group was much wider than the Idea Units. The results show that the Experienced Group of interpreters delivered ST Essential Idea Units more closely and accurately to TT renditions than the Novice Group. The performance difference by interpreters with different expertise levels could be explained by their ability to allocate cognitive resources for information processing and thus process information more quickly and accurately. In this regard, the selection of relevant Idea Units as manifested by the percentage of Essential Idea Units correctly delivered provides evidence that this is one of the main expertise areas of interpreters.

\section{Effects of Topic Knowledge vs. Working Memory Performance on Delivery of Information}

This study begins on the assumption that two qualities of the interpreters i.e. topic knowledge and working memory capacity, will have implications for the performance of interpreters during SI. Interpreters' extra-, intra- and interlingusitic knowledge helps them to overcome ST difficulties. This capacity can be represented as a continuum starting with individuals who are new to the profession and unfamiliar with the topic being discussed, through interpreters who have some professional experience and familiarity with the 
subject, to experienced interpreters who have sufficient knowledge. Thus, the working memory capacity and topic knowledge measurements of the Novice and Experienced Groups of Interpreters are compared according to their transfer of Idea Units.

The results show that there is a higher correlation between ability to transfer Idea Units and their Working Memory Span compared to Topic Knowledge among the Novice Group of interpreters. As for the Experienced Group, the interpreters' ability to transfer Idea Units did not appear to reflect higher correlation with Topic Knowledge or Working Memory Span. As for the Essential Idea Units, the Experienced Group showed a higher correlation between the delivery of Essential Idea Units and Topic Knowledge compared to the Novice Group. As mentioned, the Experienced Group of interpreters showed a higher percentage in delivery of information in ST to TT compared to the Novice Group. In particular, the Experienced Group showed much higher percentage of accuracy in delivering Essential Idea Units that were difficult i.e. which required a great deal of Topic Knowledge as well as the ability to allocate necessary working memory capacity.

Based on the findings, it can be concluded that there is some implication for working memory span and topic knowledge on the interpreter's capacity to transfer information during SI. This may be an indication that both Novice Group and Experienced Group of interpreters utilize their knowledge of the topic being discussed actively in the process of comprehension during SI. The chart below shows the correlation between working memory, knowledge about topic and experience all combined together with the interpreters' capacity to transfer information. Between the two groups of interpreters, Experienced Group of Interpreters appear to have higher correlation between their capacity to transfer information with the measurements of working memory span, topic knowledge and years of experience. However, the disparity between the two groups is not very significant. 


\section{Discussion}

It is widely thought that simultaneous interpreters simply follow what the speakers say and occasionally check for the overall logic of the speech. It is argued in this study that SI is much more than such. The interpreter will go through comprehension and analysis as well as decision making processes and exert efforts to most effectively deliver the message in the audience friendly manner. Sometimes, simultaneous interpreters will exert information and make renditions more explicit or implicit. In doing such, it is argued and shown through experiments that interpreters make use of their topic knowledge and the training to more efficiently utilize working memory capacity contribute to their performances. It is argued in this study that such endeavors or efforts on the part of the interpreters may be described as the expertise of simultaneous interpreters.

Until now, the attempt to tackle the issue of re-expression, had been mostly conducted via reports on questionnaire-based empirical research, focusing on the user's point of view (Kurz 1993; Mack \& Cattaruzza 1995). Others adopted the position of the interpreter, or examined ways in which the intrinsic constraints on performance can be taken into account. This study was an attempt to shed light on the process itself by investigating what is taking place from the interpreter's stance. The information contents of the interpreter's target text have been examined and quantified as factors contributing to the quality or acceptability of the interpreter's product. Interpreting experiment sessions to look into the mind of the interpreter and what kind of constraints they go through helped in gaining an in-depth understanding on the process of SI itself as well as the constraints and expertise involved in this mode of communication. 
110 Working Memory Performance

\section{References}

Barik, H.C. 1975. Simultaneous Interpretation: Qualitative and Linguistic Data. Language and Speech 18, 69-93.

Carpenter, P.A., et al. 1994. Working Memory Constraints in Comprehension: Evidence from Individual Differences, Aphasia, and Aging. In M.A. Gernsbacher (ed.), Handbook of Psycholinguistics. San Diego, CA: Academic Press.

Daneman, M., \& Carpenter, P.A. 1980. Individual Differences in

Working Memory and Reading. Journal of Verbal Learning and Verbal Behavior 19, 450-466.

Dillinger, M. 1994. Comprehension During Interpreting: What Do Interpreters Know that Bilinguals Don't? In S. Lambert \& B. Moser-Mercer (Eds.), Bridging the Gap: Empirical Research in Simultaneous Interpretation 155-190. Amsterdam: John Benjamins.

Gerver, D. 1975. A Psychological Approach to Simultaneous Interpretation. META 20, 119-128.

Gile, D. 1995. Basic Concepts and Models for Interpreter and Translator Training. Amsterdam: John Benjamins.

Jones, R. 1998. Conference Interpreting Explained. Manchester, UK:

St. Jerome Publishing.

Just, M.A., \& Carpenter, P.A. 1992. A Capacity Theory of Comprehension: Individual Differences in Working Memory. Psychological Review 99, 122-149.

Seleskovitch, D. 1978. Interpreting for International Conferences, Washington: Pen and Booth.

Seleskovitch, D. \& Lederer, M. 1989. A Systematic Approach to Teaching Interpretation. Trans. by J. Harmer. Luxembourg: Didier Erudition.

Setton, R. 1999. Simultaneous Interpretation, A Cognitive-Pragmatic Analysis. Amsterdam \& Philadelphia: John Benjamins. 